\title{
Sawtooth control and the interaction of energetic particles
}

\author{
I.T. Chapman ${ }^{1}$, V.G. Igochine ${ }^{2}$, J.P. Graves ${ }^{3}$, S.D. Pinches ${ }^{1}$, A. Gude ${ }^{2}$, \\ I. Jenkins ${ }^{1}$, M. Maraschek ${ }^{2}$, G. Tardini ${ }^{2}$, the ASDEX Upgrade Team and \\ JET EFDA Contributors ${ }^{\mathrm{a}}$ \\ ${ }^{1}$ EURATOM/UKAEA Fusion Association, Culham Science Centre, Abingdon, Oxfordshire OX14 3DB, UK \\ ${ }^{2}$ MPI für Plasmaphysik, EURATOM-Association D-85748 Garching, Germany \\ ${ }^{3}$ CRPP, Association EURATOM-Confédération Suisse, EFPL, Lausanne, Switzerland \\ E-mail: ian.chapman@ukaea.org.uk
}

Received 1 December 2008, accepted for publication 2 February 2009

Published 25 February 2009

Online at stacks.iop.org/NF/49/035006

\begin{abstract}
Long-period sawteeth have been observed to result in the low- $\beta$ triggering of neo-classical tearing modes (NTMs), which can significantly degrade plasma confinement. In ITER, the stabilizing effects of the fusion-born $\alpha$ particles are likely to exacerbate this. Consequently, in order to avoid triggering NTMs, many techniques have been proposed to control, and in particular, to destabilize the sawtooth oscillations. Here, sawtooth behaviour in off-axis NBI-heated plasmas in JET and ASDEX Upgrade is presented. It is found that the energetic particles born outside the $q=1$ surface due to off-axis NBI can destabilize the sawteeth, even in the presence of stabilizing on-axis fast particles. In order to model the stability of the $n=1$ internal kink mode, which is associated with the sawtooth oscillations, both a magnetohydrodynamic code including toroidal rotation and a drift kinetic code have been employed. The modelling highlights the significant role played by both the passing energetic particles and the toroidal flow shear in determining the kink mode stability in the presence of an energetic particle population.
\end{abstract}

PACS numbers: $52.35 \mathrm{Py}, 52.55 \mathrm{Fa}, 52.55 \mathrm{Pi}, 52.55 \mathrm{Tn}$

(Some figures in this article are in colour only in the electronic version)

\section{Introduction}

Developing methods to control sawtooth oscillations remains a key area of research for high performance operation of tokamak plasmas. Whilst the plasma typically survives the small drops in core temperature and density caused by sawteeth, the coupling of sawteeth to other magnetohydrodynamic (MHD) modes is of serious concern. It has been shown that plasmas that exhibit long sawtooth periods are more susceptible to neo-classical tearing modes (NTMs) [1,2]. Furthermore, in burning plasmas, such as those in ITER, the fusion-born $\alpha$ particles are predicted to lead to long sawtooth periods [3-5], meaning that the plasma is likely to be more susceptible to confinement-degrading NTMs. Consequently, many techniques have been developed to destabilize sawteeth in an attempt to avoid triggering NTMs, whilst retaining the benefits of small, frequent sawtooth crashes, such as the prevention of core impurity accumulation [6].

Porcelli et al [3] developed a model of linear stability thresholds to determine when a sawtooth crash can occur. The

a See the appendix of F. Romanelli et al 2008 Proc. 22nd Int. Conf. on Fusion Energy 2008 (Geneva, Switzerland, 2008) (Vienna: IAEA). fundamental trigger of the sawtooth crash is the onset of an $m=$ $n=1$ mode. The dynamics of this instability are constrained by many factors including not only the macroscopic drive from ideal MHD, but collisionless kinetic effects related to high energy particles [7-9] and thermal particles [10], as well as non-ideal effects localized in the narrow layer around $q=1$. In heated plasmas, there are essentially two relevant sawtooth triggering criteria [3]:

$\pi \frac{\widehat{\delta W}}{s_{1}}<\hat{\rho} \quad$ and $\quad s_{1}>s_{\text {crit }}(\beta)$

(Resistive two-fluid instability),

$\pi \frac{\widehat{\delta W}}{s_{1}}<-\frac{\omega_{* \mathrm{i}} \tau_{\mathrm{A}}}{2}$

(Ideal instability),

where $\tau_{\mathrm{A}}=\sqrt{3} R_{0} / v_{\mathrm{A}}$, the Alfvén speed is $v_{\mathrm{A}}=$ $B_{0} / \sqrt{\mu_{0} n_{0} M_{0}}, \hat{\rho}=c_{\rho} \rho / \kappa_{1}^{1 / 2} r_{1}, \rho$ is the ion Larmor radius, $n$ is the plasma density, $M$ is the ion mass, $c_{\rho}$ is a constant of order unity, $\omega_{* i}$ is the ion diamagnetic frequency, $\kappa_{1}$ is the elongation of the $q=1$ surface, $r_{1}$ is the radius of the $q=1$ surface and $s_{1}=r /\left.q(\mathrm{~d} q / \mathrm{d} r)\right|_{q=1}$ is the magnetic shear 
at the $q=1$ surface. Consequently, long sawteeth can be destabilized (i.e. a crash can be triggered) by enhancing $s_{1}$ (through localized current drive, for example), or through $\delta W$ reduction or reversal.

For instance, current driven by electron cyclotron resonance heating has been used to control sawtooth periods in ASDEX Upgrade [11], TCV [12], JT-60U [13] and TEXTOR [14]. Furthermore, recent initial results in TORE-SUPRA [15] suggest that electron cyclotron current drive (ECCD) can be used to destabilize long-period sawteeth stabilized by a population of fast ions resulting from simultaneous ion cyclotron resonance heating (ICRH). It has also been shown that off-axis ICRH can be used to destabilize fast ion induced long sawteeth [1, 16-18].

Whilst ICRH and ECCD sawtooth control actuators are envisaged for ITER, results from plasmas heated using neutral beam injection have helped to exemplify the physical processes that determine sawtooth behaviour. For example, shorter sawtooth periods than those in Ohmically heated plasmas can be achieved in NBI-heated plasmas in JET [19, 20], MAST [21] and TEXTOR [22]. Each experiment exhibits an asymmetry of sawtooth period with respect to NBI direction. In order to understand sawtooth stabilization, the interaction of MHD and fast particle effects must be considered. In MAST, the asymmetric stabilization of sawteeth by NBI heating has been explained in terms of the direction of the strong toroidal flows induced by the NBI, relative to the ion diamagnetic drift [21]. Whilst fast ions do have a stabilizing influence, the significant trapped fraction in spherical tokamaks is stabilizing in either co- or counter-NBI regimes, meaning that kinetic effects cannot explain the experimentally observed minimum in sawtooth period. However, in JET, the toroidal rotation is significantly smaller, and the sawtooth behaviour can only be explained by the effects of the fast ions. The sawtooth period is observed to vary in the same way as in MAST, with lengthening period as the co-NBI power increases, and a minimum in sawtooth period in the counter-NBI regime [19]. This minimum occurs because (i) the counter-passing ions give a strongly destabilizing contribution and (ii) the flow shear in JET reduces the stabilizing effect of the trapped ions injected counter- $I_{\mathrm{p}}$ [20]. In TEXTOR, the sawtooth period reaches a maximum in the counter-NBI regime due to a competition between the gyroscopic stabilization of the kink mode and the destabilization arising in the presence of counter-passing fast ions.

Following the explanation of these on-axis NBI experiments together with experiments in JT-60U, which showed that passing fast ions from negative-ion NBI could also influence sawtooth behaviour [23], it was proposed [7, 24, 25] that NBI could also be used to control sawteeth. Subsequently, experiments in JET [5] have shown that off-axis NBI results in short sawtooth periods provided that the deposition location of the peak of the fast ion distribution is outside the $q=1$ radius. Furthermore, in order to demonstrate the suitability of off-axis co-NBI as a sawtooth control actuator, it has been shown that its application is able to result in destabilization of otherwise strongly stabilized sawteeth (arising from on-axis NBI fast ions).

Section 2 reports on sawtooth behaviour in off-axis NBI-heated plasmas in JET and ASDEX Upgrade. These experimental results are then modelled using the codes described in section 3. The effect of the passing fast ions and toroidal flow shear is also investigated in section 3 together with a comparison of passing ion effects to analytic theories. In section 4 we report on the linear modelling of the stability of the $n / m=1 / 1$ internal kink mode in ASDEX Upgrade. Finally, the implication of these results together with a discussion of how sawtooth control may be achieved in ITER is presented in section 5 .

\section{Sawtooth behaviour in off-axis NBI-heated plasmas}

\subsection{JET}

In [5] the possibility of using off-axis NBI as a sawtooth control actuator has been investigated experimentally and through stability analyses. Experiments in JET have shown that sawtooth oscillations are considerably more unstable when the plasma is heated with co-directed off-axis NBI than in on-axis NBI regimes. Furthermore, the application of NBI heating deposited off-axis can destabilize sawteeth which had previously been strongly stabilized by concurrent on-axis NBI heating. This is explained qualitatively through the rôle of the passing ions in determining the stability of the $n / m=$ $1 / 1$ internal kink mode. In JET the sawtooth behaviour is dominated by fast ion effects as the off-axis neutral beam current drive is weak and broadly deposited.

In the experiments reported in [5], the total beam power is kept constant when the off-axis power is applied in order to keep the fast ion $\beta$ the same, and so not affect the ideal mode stability. However, in order to demonstrate the suitability of off-axis co-NBI as a control tool, it is necessary to show whether ancillary application of off-axis beams is able to result in destabilization of otherwise strongly stabilized sawteeth. Such sawtooth control has been demonstrated in JET using off-axis ICRH to shorten the sawtooth period when a co-existing fast ion population exists in the core due to concurrent ICRH heating with a different phasing [17]. However, this actuator is very strongly dependent upon the precise location of the deposition of fast ions, making it difficult to control. By applying on-axis NBI throughout the discharge in order to stabilize the sawteeth, the sawtooth behaviour under simultaneous application of off-axis NBI is an appropriate test of the use of off-axis beams as a sawtooth control mechanism [5]. Figure 1 shows the beam time traces and soft x-ray emission from JET discharge 58855. The sawtooth period is substantially lengthened during the on-axis only phase ( $\sim 315 \mathrm{~ms}$ ) before decreasing to approximately the period of Ohmic sawteeth when the off-axis power is applied $(\sim 120 \mathrm{~ms})$ but the total applied power is held constant. This clear destabilization of the sawteeth when off-axis NBI is applied is also demonstrated in other JET discharges [5]. Furthermore, if the sawtooth behaviour is compared between $16-18 \mathrm{~s}$ and $20-22 \mathrm{~s}$ when there is constant on-axis power, then it is evident that the additional application of off-axis NBI can be used to destabilize long sawteeth. The sawtooth period decreases by a factor of 2 when the off-axis NBI is applied, even though $\beta_{h}$-the fast ion pressure-increases. 


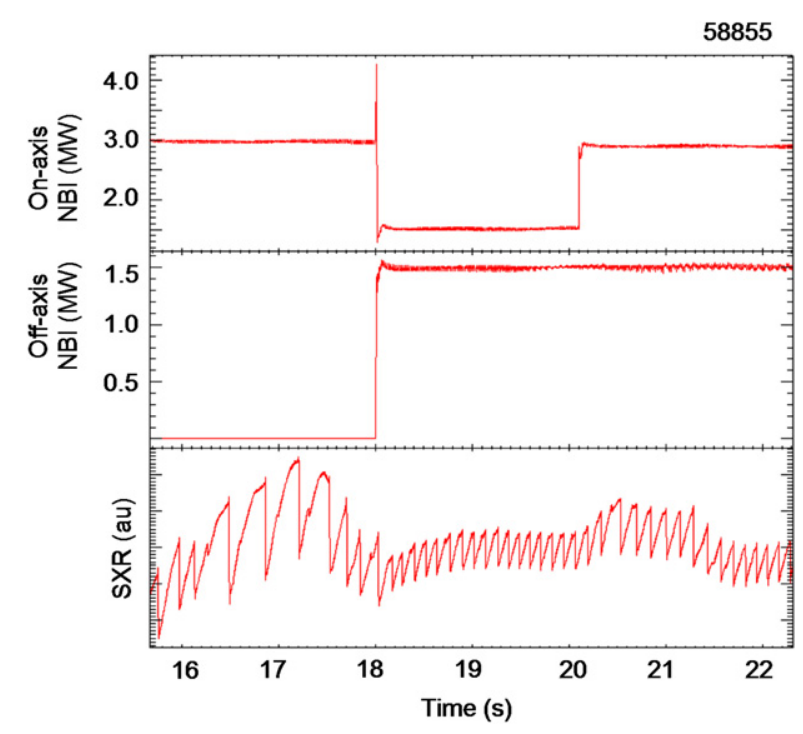

Figure 1. The SXR emission and beam heating waveforms for JET shot 58855 . The sawtooth period is significantly shorter when the total $\beta_{h}$ is kept constant, but some off-axis NBI is used in place of on-axis heating. Further, this discharge also shows that the application of ancillary off-axis NBI can decrease the sawtooth period, despite an overall increase in $\beta_{h}$.

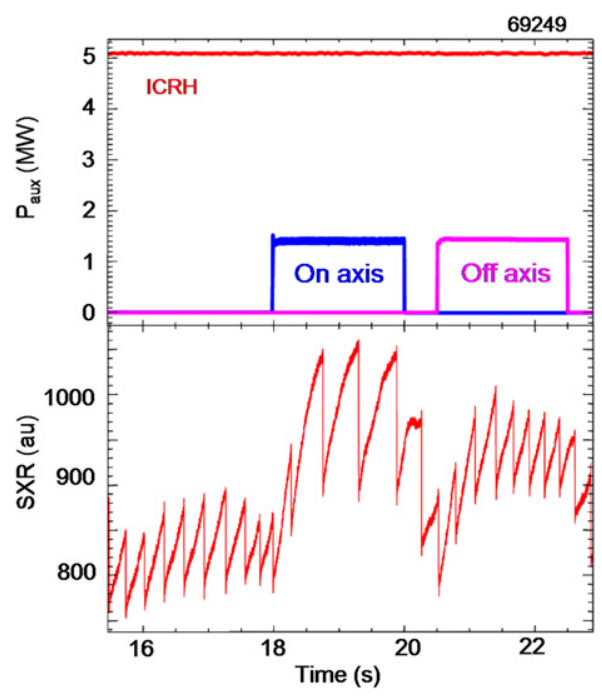

Figure 2. The SXR emission trace for JET discharge 69249 together with the auxiliary heating waveforms. The sawteeth are significantly shorter during the off-axis NBI heated phase, than when on-axis NBI is applied.

In order to further test the applicability of neutral beam injection for sawtooth control, we have applied off-axis NBI in JET plasmas also heated with ICRH. The ICRH is used to heat the plasma in order to generate a more isotropic population of highly energetic particles in the core than is achieved with NBI heating, representative of the fusion-born $\alpha$ particles expected in ITER. Figure 2 shows JET discharge 69249, which is heated with $5 \mathrm{MW}$ of ICRH power throughout, with $2 \mathrm{~s}$ pulses of NBI power. When the on-axis NBI is applied, the sawtooth period increases by more than a factor of 2 , whilst the off-axis NBI leads to approximately the same sawtooth period as the RF-only phase. Whilst the off-axis NBI does not result in a strong stabilization as exhibited with the core heating, it does

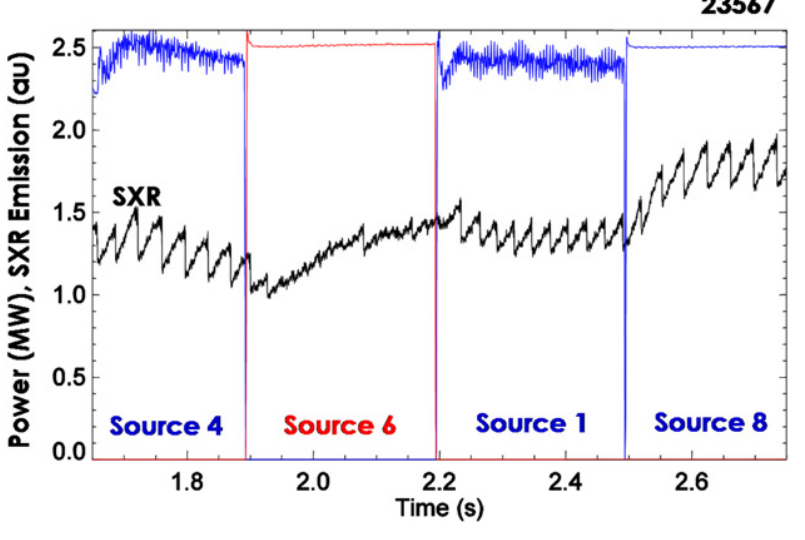

Figure 3. The influence of different neutral beam injection sources on sawtooth behaviour in ASDEX Upgrade discharge 23567.

Sources 1, 4 and 8 are on-axis and source 6 is directed off-axis.

not seem to destabilize the RF-induced long sawteeth either. However, it is plausible that destabilization could occur if the deposition location of the peak of the fast ion distribution was in the right place with respect to the $q=1$ surface, as discussed in the modelling presented in section 4 .

\subsection{ASDEX Upgrade}

ASDEX Upgrade is ideally suited to investigate the potential role of off-axis NBI for sawtooth control because it has two tangential NBI sources, which inject fast ions towards the midradius of the plasma. Furthermore, it is also equipped with an ICRH system, which can be used to generate an energetic particle population in the core, like that expected in a burning plasma. ASDEX Upgrade has two neutral beam injector boxes, each equipped with four positive ion neutral injectors (PINIs). The sources differ in tangential and radial injection, meaning that the energetic particle populations born in the plasma due to the different PINIs will differ in both trapped fraction and deposition radius. The geometry of the ASDEX Upgrade NBI heating system and the tangency radius of each source can be found in [11].

The sawtooth behaviour in a plasma heated with both on-axis and off-axis neutral beams is compared in figure 3 . Just as in reference [11], the sawtooth period is approximately constant during the different on-axis NBI phases (sources 1, 4 and 8 ), whereas there is distinctly different behaviour when heating with off-axis source 6 .

In order to determine whether neutral beam injection can be used as a sawtooth control tool, off-axis NBI has been applied in ASDEX Upgrade discharges which have ICRH fast ion stabilized sawteeth. In discharges 23476 and 23477, the plasma is first heated with $4.5 \mathrm{MW}$ of ICRH power, leading to sawteeth in the presence of core fast ions. Then the most tangential off-axis PINI source is applied at the same time as the ICRH in an attempt to destabilize the sawteeth. Figure 4 shows the sawtooth behaviour in discharge 23476, with plasma current $I_{\mathrm{p}}=1 \mathrm{MA}$, toroidal magnetic field $B_{\mathrm{T}}=2.5 \mathrm{~T}$ and density $n_{\mathrm{e}} \approx 9 \times 10^{19} \mathrm{~m}^{-2}$. Clearly the sawtooth period increases from approximately $45 \mathrm{~ms}$ in the ICRH only phase, to $\tau_{\mathrm{s}} \sim 55 \mathrm{~ms}$ when the ancillary off-axis NBI is applied. The current density profile has been calculated 


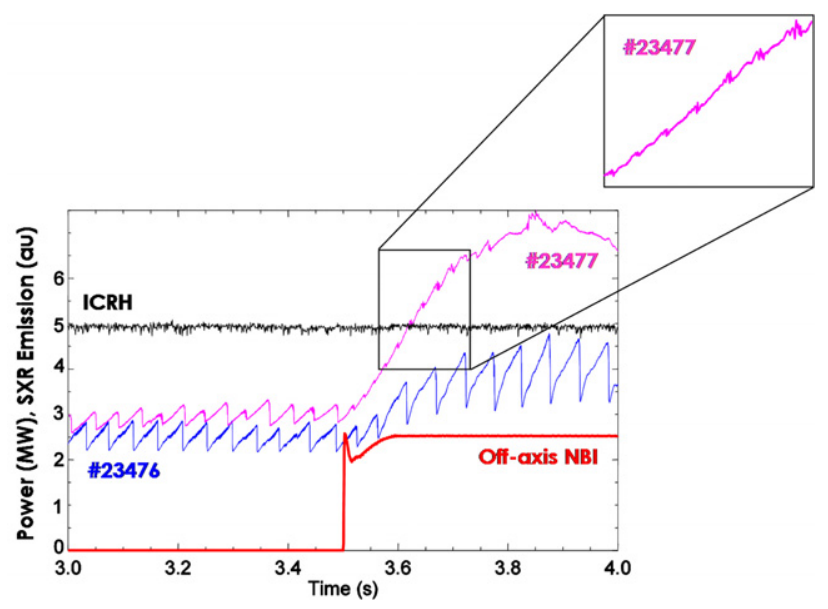

Figure 4. The effect of off-axis NBI on sawteeth in ASDEX Upgrade plasmas simultaneously heated with ICRH. In shot 23476 the $q=1$ surface is broad, and the off-axis NBI increases the sawtooth period. In contrast, in shot 23477 , where $q_{0}$ is higher, the sawtooth behaviour is significantly altered by the off-axis NBI.

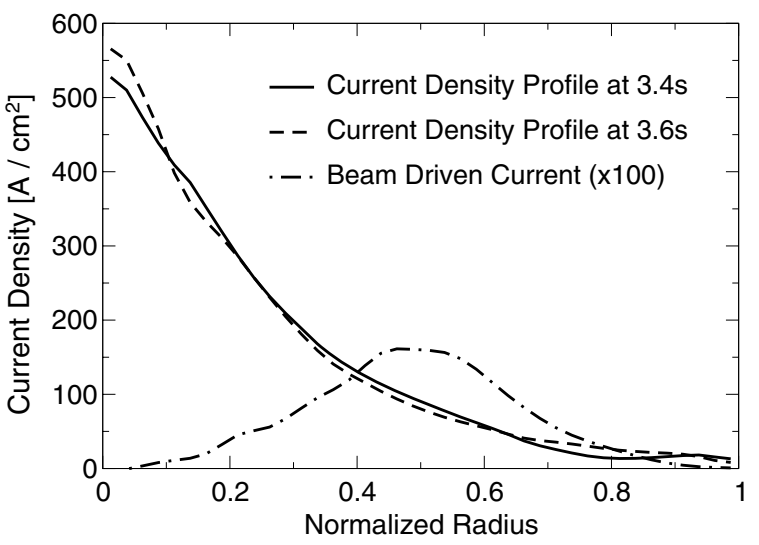

Figure 5. The current density profile for ASDEX Upgrade discharge 23476 calculated by TRANSP. The neutral beam driven current is very broad and small compared with the plasma current (here magnified by two orders of magnitude).

using the TRANSP transport code [26]. Although the neutral beam current drive does result in a small perturbation to the current density profile, as illustrated in figure 5, the $q$-profile does not change significantly. Indeed, the inversion radius found from the soft $\mathrm{x}$-ray emission does not change when the off-axis NBI is applied, indicating that the $q$-profile has not altered significantly.

Figure 4 also shows the sawtooth behaviour in discharge 23477 where the toroidal field is raised to $B_{\mathrm{T}}=2.7 \mathrm{~T}$ in order to move the $q=1$ surface radially inwards. Whilst the difference in the magnetic field means that the radial location of the ICRH resonance shifts towards the low-field side, the sawtooth period in the ICRH-only phase does not change significantly between shot 23476 and 23477 . However, it should be noted that $\beta_{\mathrm{N}}$ is approximately $25 \%$ lower in shots 23477 than in 23476 . This cannot be explained entirely by the increase in toroidal field, so presumably the ICRF heating is slightly degraded when the resonance is shifted. When the off-axis NBI is applied in shot 23477, the sawteeth seem to become very small and their frequency doubles. However, it is not clear whether the sawtooth oscillations become small or disappear entirely. TRANSP calculations indicate that the neutral beam current drive is small and very broad and it is not anticipated to significantly alter the $q$-profile.

In both discharges the soft $\mathrm{x}$-ray emission increases when the off-axis NBI is applied. Within the error bars, the density profile is unaltered by the off-axis NBI present at $3.6 \mathrm{~s}$ compared with the case without NBI heating at $3.4 \mathrm{~s}$. However, in both discharges the temperature profile is increased by approximately $20 \%$ due to neutral beam heating. This change in the temperature does not account for the significant increase in the soft x-ray emission in discharge 23477 indicated in figure 4. One possible explanation is that when the sawteeth are sufficiently small and benign a transport barrier can be established and the impurities become peaked in the core, resulting in the increased emission. However, it should be noted that there is no indication of an internal transport barrier in the electron temperature data, and unfortunately there were no measurements of the ion temperature due to the design of these discharges. Alternatively, it could be argued that the impurity peaking arises because the sawteeth have disappeared entirely and so the absence of any redistribution of the core plasma allows impurity accumulation.

Modelling to assess the stability of the $n=1$ kink mode in these ASDEX Upgrade plasmas is presented in section 4. Studies to assess the effect of moving the NBI fast ion population with respect to the $q=1$ surface by tilting an off-axis PINI will be reported elsewhere.

\section{Modelling tools}

In order to make an assessment of the stability of the $n=1$ internal kink mode, and how this may influence when a sawtooth crash occurs in accordance with the model presented in [3], the change in the potential energy of the mode, $\delta W$, must be calculated. The MHD part, $\delta W_{\text {MHD }}$, is calculated using the MishKA-F code [29]. MishKA-F uses an equilibrium generated by the fixed-boundary HELENA equilibrium code [30], which solves the static Grad-Shafranov equation. However, it is important to note that rotational modification of the equilibrium can affect the stability of the $n=1$ internal kink mode $[28,31]$. Whilst the rotation effects are included in MishKA-F in an inconsistent manner, this is an acceptable approximation for the low rotation and equilibrium profiles considered in this paper. The effects of equilibrium profiles and the validity of an inconsistent equilibrium flow treatment will be considered in a future publication.

The kinetic contribution, $\delta W_{h}$, is then quantified using the drift kinetic HaGIS code [32]. The input required by HaGIS consists of the equilibrium (supplied from the Helena code), the perturbation (calculated by MishKA-F) and the initial fast particle distribution function (which can be calculated by a Monte-Carlo transport code, like TRANSP [26]). This chain of code execution is illustrated in figure 6 .

When the equilibrium pressure profile is supplied to Helena, it contains the fast ion pressure but assumes that the fast ion population is isotropic. Strictly, the equilibrium should include anisotropic effects, as in [33], since the fast ions modify the equilibrium in a complicated way due to the fact that the fast ion pressure components are no longer flux surface quantities. 


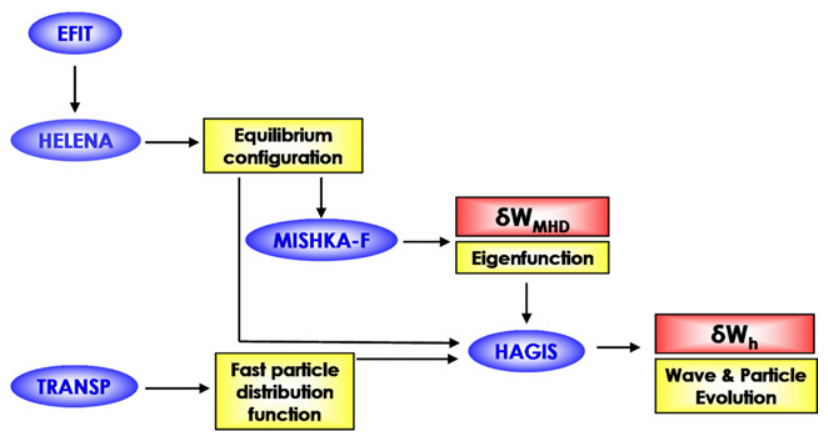

Figure 6. The numerical modelling tools used to assess the role of energetic particles and toroidal rotation upon sawtooth stability.

Consequently, the $\delta W_{h}$ produced by HaGis must be reduced by the contribution from isotropic adiabatic particles, $\delta W_{h f}^{\text {iso }}$, with the same $\beta_{h}$ as those which are retained in the $\delta W_{\text {MHD }}$ calculation in MisHKA-F.

\section{1. $\delta W_{h}$ calculation}

In order to quantify the effect of the energetic particles upon the $n=1$ internal kink mode, it is necessary to calculate the change in the potential energy of the mode, $\delta W_{h}$, in accordance with [34, 35]:

$\delta W_{h}=\frac{1}{2} \int \mathrm{d} \Gamma\left(m v_{\|}^{2}+\mu B\right) \delta f \sum_{m} \kappa \cdot \boldsymbol{\xi}^{(m) *}(r, t) \mathrm{e}^{-\mathrm{i}(\omega t+n \zeta-m \theta)}$,

where $\theta$ is the poloidal angle, $\boldsymbol{\kappa}=\boldsymbol{b} \cdot \nabla \boldsymbol{b}$ is the magnetic curvature vector, $\boldsymbol{b}=\boldsymbol{B} / \boldsymbol{B}$ and $\mathrm{d} \Gamma$ is an infinitessimal volume element of phase-space. In order to relate the displacement to the electric scalar potential, $\Phi$, and the vector potential, $\boldsymbol{A}$, which is how it is expressed in HaGIs, we use

$$
\boldsymbol{v}^{*}=\frac{\mathrm{d} \boldsymbol{\xi}^{*}}{\mathrm{~d} t}=\frac{\boldsymbol{E}^{*} \times \boldsymbol{B}}{|B|^{2}}
$$

Since the displacement is assumed to be of the form $\xi \sim$ $\xi_{0} \mathrm{e}^{-\mathrm{i} w t}$, then equation (4) becomes

$$
\boldsymbol{\xi}^{*}=-\frac{\boldsymbol{E}^{*} \times \boldsymbol{B}}{\mathrm{i} \omega|B|^{2}}
$$

Since $\boldsymbol{E}^{*}=\partial \boldsymbol{A}^{*} / \partial t-\nabla \Phi^{*}$, and in HaGIS it is assumed that $\boldsymbol{A}=\alpha \boldsymbol{B}$, then

$$
\begin{aligned}
\boldsymbol{E}^{*} \times \boldsymbol{B} & =\left(\frac{\partial \boldsymbol{A}^{*}}{\partial t}-\nabla \Phi^{*}\right) \times \boldsymbol{B} \\
& =\left(\frac{\partial \alpha^{*} \boldsymbol{B}}{\partial t}-\nabla \Phi^{*}\right) \times \boldsymbol{B} \\
& =-\nabla \Phi^{*} \times \boldsymbol{B} .
\end{aligned}
$$

Substituting this into equation (5) yields

$$
\xi^{*}=\frac{\nabla \Phi^{*} \times \boldsymbol{B}}{\mathrm{i} \omega|B|^{2}} .
$$

Now, using equation (7) and the fact that $\boldsymbol{\kappa}=\boldsymbol{b} \cdot \nabla \boldsymbol{b}=$ $(\nabla \times \boldsymbol{b}) \times \boldsymbol{b}$, equation (3) can be rewritten as

$\delta W_{h}=-\frac{1}{2} \int \mathrm{d} \Gamma\left(m v_{\|}^{2}+\mu B\right) \delta f \boldsymbol{b} \times(\nabla \times \boldsymbol{b}) \cdot \frac{\nabla \Phi^{*} \times \boldsymbol{b}}{\mathrm{i} \omega|B|}$.
Finally, using $\nabla \Phi_{\perp}^{*}=\nabla \Phi^{*}-\left(\boldsymbol{b} \cdot \nabla \Phi^{*}\right) \boldsymbol{b}$, equation (8) can be rewritten to give the expression for the change in the potential energy of the mode calculated in HAGIS:

$$
\delta W_{h}=\frac{1}{2 \mathrm{i} \omega} \int \mathrm{d} \Gamma \frac{\left(m v_{\|}^{2}+\mu B\right)}{|B|} \delta f \nabla \Phi_{\perp}^{*} \cdot \nabla \times \boldsymbol{b} .
$$

By summation for all particles, $\delta f$ can be found and thus $\delta W_{h}$ can be calculated according to equation (9). The $\delta W_{h}$ calculated here is a collisionless correction to the MHD response. Equation (9) does not include compressibility as the HAGIS code assumes that the fast ions are collisionless. In modern large, hot tokamaks, the fast ion temperatures are sufficiently high that the effective collision frequency is much smaller than diamagnetic frequency, $v^{\mathrm{i}} / \omega_{* \mathrm{i}} \ll 1$, thereby justifying the collisionless limit. In the collisionless limit of drift kinetic theory, the parallel flow kinetic equation is replaced by the assumption that the perturbations are incompressible. In the modelling presented here the growth rate of the mode is sufficiently small that this is appropriate. The plasma compressibility can still be included in the calculation of the MHD drive for the mode with MishKA-F, where it is assumed that the adiabatic index $\gamma=5 / 3$. Whilst compressibility effects may play some role [36] in determining $\delta W_{h}$, sawtooth stability theory [3] assumes that the fast ions can be considered as collisionless, and has had notable empirical success $[20,37]$.

The calculation of $\delta W_{h}$ in HAGIS is a linear result with no time evolution information. In order to calculate the change in the potential energy of the wave in the presence of an energetic particle population, the perturbation and the distribution function must be changed iteratively until the wave potential energy converges.

No direct comparison can be drawn between the modelling and the experimental results since the sawtooth behaviour is inherently non-linear, whereas here we consider the linear stability of the $n=1$ kink mode. In order to fully explain the sawtooth behaviour exhibited experimentally, a transport code would need to be coupled to the accurate evaluation of the $\delta W_{h}$ terms presented here and all terms evolved in time. It is, however, very plausible that the lower $\delta W_{h}$ does directly produce shorter sawteeth, although the modelling presented here is only qualitative with respect to comparison with experiment.

\subsection{Passing particle stabilization mechanism}

Previous modelling of JET NBI-heated plasmas has concentrated primarily on the effects of the trapped fast particles [37]. However, the neutral beam heating in JET gives rise to a predominantly passing population. Recent analytic theory $[7,38,39]$ has suggested that the co-passing energetic particles can stabilize the $1 / 1$ internal kink mode whereas counter-passing fast ions can have a destabilizing influence. It is predicted that the effect of the passing fast ions is determined by the finite orbit width term contained within $\delta W_{h}^{p} \sim \int_{0}^{r_{1}}\left(\vec{\xi} \cdot \nabla\left\langle P_{h}\right\rangle\right)(\vec{\xi} \cdot \vec{\kappa}) \mathrm{d} \vec{r}$, where $P_{h}$ is the hot particle pressure. As illustrated in figure 9 of [5], this means that on-axis co-passing and off-axis counter-passing ions will be stabilizing whereas off-axis co-passing and on-axis counterpassing ions will be destabilizing. These predictions have 


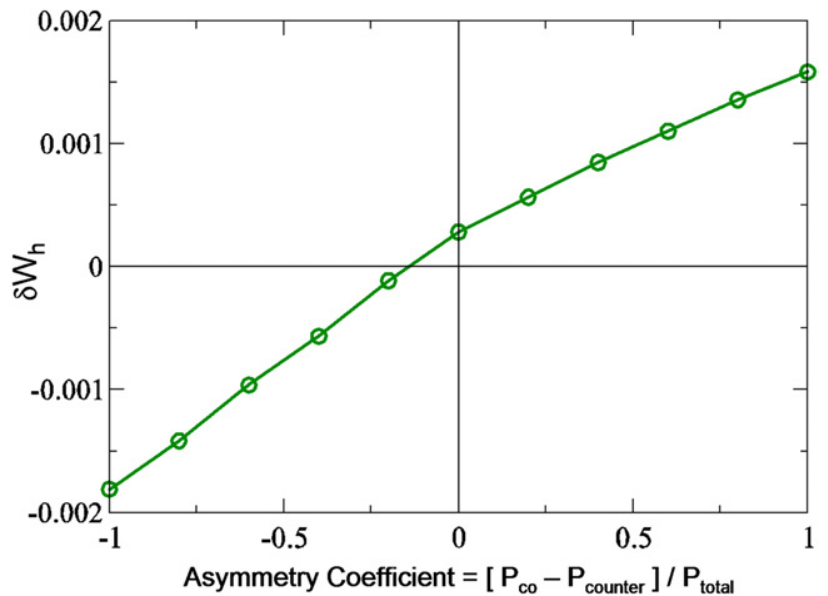

Figure 7. The contribution to $\delta W_{h}$ from the passing fast ions when the distribution function is scanned from entirely co-passing (asymmetry coefficient $=1$ ) to entirely counter-passing $(-1)$.

been experimentally verified on JET [5,20] and JT-60U [23] where the toroidal flow is slow enough that the kinetic effects dominate the sawtooth behaviour. These effects have helped to explain the experimental results observed in JET [19,20] and TEXTOR [22] exhibiting asymmetric sawtooth behaviour in co- or counter-NBI-heated plasmas. The local effects of the passing energetic ions are often found to dominate over the effects of the trapped fast ions. The effect of the asymmetric passing ions can be enhanced when the effective orbit width becomes large. This is the case when the ions have large thermal velocity or when the distribution has a large fraction of barely passing ions.

In order to compare the effect of co- and counter-passing on-axis fast ions on the internal kink mode, we use a simple model equilibrium and vary the fast ion distribution function from entirely on-axis co-passing to entirely counter-passing. The equilibrium used has a pressure profile, $\mathrm{d} p / \mathrm{d} \psi=p^{\prime}=$ $p^{\prime}(0)(1-\hat{\psi})$ and a current profile, $\langle j\rangle=j(0)(1-\hat{\psi})$, where $\hat{\psi}$ is normalized poloidal flux. The plasma has a circular boundary, an aspect ratio of 10 , safety factor on-axis, $q_{0}=0.8$ and poloidal beta, $\beta_{\mathrm{p}}=0.3$. The equilibrium is static and unstable to the ideal $n=1$ internal kink mode. Figure 7 shows the contributions to $\delta W_{h}$ from the passing ions with respect to the degree of asymmetry of the distribution function. In this case, the asymmetry coefficient, $C_{\lambda}=\left(P_{\text {co }}-P_{\text {counter }}\right) /\left(P_{\text {co }}+P_{\text {counter }}\right)$, is unity when the fast ion population is assumed to consist entirely of co-passing ions, whilst $C_{\lambda}=-1$ when an ideal counter-passing beam of fast ions is assumed. When $C_{\lambda}=0$, balanced beams are assumed, with $\beta_{h}$ constant throughout this scan. When the distribution is entirely co-passing, the fast ions have a strongly stabilizing effect on the internal kink mode. Conversely, the contribution to $\delta W_{h}$ from purely counter-passing ions is strongly destabilizing. When the passing ions are balanced, the fast ions have a weakly stabilizing effect on the kink mode, due to higher order terms. The passing ions provide a contribution to the change in the potential energy of the $n=1$ kink mode of the form

$\delta W_{h}^{p} \sim \int \frac{\omega-\omega_{*}}{\omega-\left\langle\omega_{\mathrm{d} h}\right\rangle-k_{\|} v_{\|}} \equiv \int \frac{\omega-\omega_{*}}{\omega-\left\langle\omega_{\mathrm{d} h}\right\rangle-(m-n q) \sigma v}$,

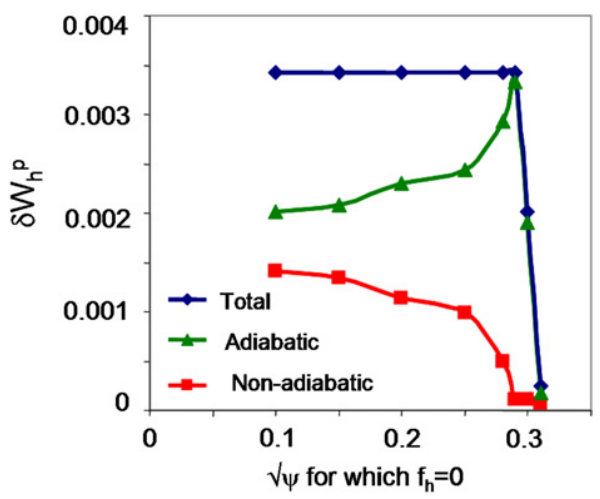

Figure 8. The contribution to $\delta W_{h}$ from the passing fast ions when the energetic particle distribution function is zeroed inside $s=\sqrt{\psi}$.

where $\sigma= \pm 1$ indicates the direction of injection and here $v=v_{\|}$. This means that in the case of balanced co- and counter- beams, the total contribution from the passing fast ions to the mode stability is

$$
\begin{aligned}
\delta W_{h}^{p} \sim & \int\left(\frac{\omega-\omega_{*}}{\omega-\left\langle\omega_{\mathrm{d} h}\right\rangle+\sigma(1-q) v}\right. \\
& \left.+\frac{\omega-\omega_{*}}{\omega-\left\langle\omega_{\mathrm{d} h}\right\rangle-\sigma(1-q) v}\right) .
\end{aligned}
$$

Assuming that $\omega \ll \omega_{*}$ and Taylor expanding equation (11) gives

$$
\begin{aligned}
\delta W_{h}^{p}= & \frac{\omega_{*}}{\sigma(1-q) v}\left[1-\frac{\left\langle\omega_{\mathrm{d} h}\right\rangle}{\sigma(1-q) v}\right] \\
& -\frac{\omega_{*}}{\sigma(1-q) v}\left[1+\frac{\left\langle\omega_{\mathrm{d} h}\right\rangle}{\sigma(1-q) v}\right] \\
= & \frac{\omega_{*}\left\langle\omega_{\mathrm{d} h}\right\rangle}{v^{2}(1-q)^{2} / R^{2} q^{2}} .
\end{aligned}
$$

This leaves a small but positive contribution from the higher order $O\left(v^{2}\right)$ terms, meaning that the balanced beams case produces a weakly stabilizing influence on the kink mode. The expression for $\delta W_{h}^{p}$ discussed here is vastly simplified and does not apply to full toroidal geometry, but is used merely to explicate the passing ion effects in the case of balanced beams. The full expression for $\delta W_{h}^{p}$ can be found in [7].

\subsection{Comparison to analytic theories}

Wang et al [39] proposed that the non-adiabatic passing ion effects arise due to the gradient $\nabla f_{h}$ integrated over the $q=1$ radius. In contrast, Graves [7] suggested that the non-adiabatic passing particle effects are counteracted by an adiabatic contribution, but that an additional adiabatic contribution survives from the fast ions which intersect the $q=1$ flux surface. This latter mechanism depends on $\partial f_{h} / \partial P_{\zeta}$ at $q=1$ only and is more sensitive to localized heating. Figure 8 shows the passing particle contribution to $\delta W_{h}$ in a typical JET discharge for a non-symmetric fast ion distribution which is Maxwellian with respect to energy and Gaussian with respect to pitch angle. The distribution function is artificially taken to be zero inside a finite radius, indicated on the $x$-axis. It is evident that when no gradient exists in a region around $q=1(s=\sqrt{\psi}=0.31)$ bounded by the orbit width, the 


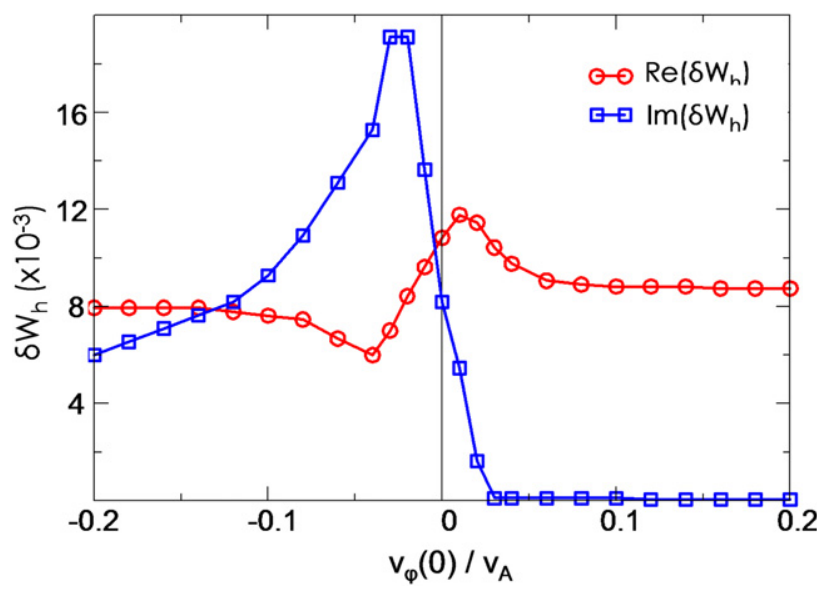

Figure 9. Effect of flow shear on trapped particle stabilization. The contribution to $\delta W_{h}$ from the non-adiabatic trapped fast ions with respect to the equilibrium velocity shear.

passing ions do not contribute to the kink mode stability, in excellent accordance with analytic theory presented in [7]. The fact that unbalanced passing ions contribute only via a radial gradient in $f_{h}$ close to $q=1$ has important implications for sawtooth control in ITER using negative NBI heating at varying deposition radii [7]. The strong contribution of the passing particles comes from ions close to the trappedpassing boundary [18] where their orbit widths, $\Delta_{b}$, are large, $\delta W_{h} \sim \Delta_{b}$.

\subsection{Including flow shear effects}

The equilibrium toroidal flow shear can also significantly affect the stabilization of the kink mode arising from the presence of energetic trapped ions [40]. Therefore, the equilibrium sheared flow has also been included in the HaGis code. Whilst plasma flows must be of the order of the ion sound speed to influence MHD stability significantly, much smaller toroidal flows can affect the kinetic contributions to the change in the potential energy of the kink mode, as long as the $\boldsymbol{E} \times \boldsymbol{B}$ rotation induced by the NBI momentum injection is radially sheared [8]. When equilibrium flow shear is introduced, it has two effects on the wave-particle interactions: firstly, in the laboratory frame, the electric potential experienced by the particles includes an extra factor induced by the local equilibrium $\boldsymbol{E} \times \boldsymbol{B}$ rotation, $\Phi^{\prime}=$ $-r B_{0} \Omega_{E} / q$. Secondly, the diamagnetic and precessional drift frequencies are Doppler shifted by the local equilibrium rotation and the mode frequency is shifted by the $\boldsymbol{E} \times \boldsymbol{B}$ rotation frequency, $\omega \rightarrow \omega-q \Phi^{\prime} / B_{0} r$.

The effect of toroidal flow shear on the stabilizing contribution from the energetic ions is modelled using HaGIS for JET discharge 60998 by prescribing the toroidal co-current rotation profile from the charge exchange diagnostic. The equilibrium and the fast ion distribution function are kept constant as the amplitude of the equilibrium flow is changed. Figure 9 shows the effect of sheared rotation on both $\Re e\left(\delta W_{h}\right)$, which quantifies the stabilizing effect of the fast ions, and $\Im m\left(\delta W_{h}\right)$, which represents the Landau energy transfer due to resonance between the trapped ions and the kink mode. In this figure, negative rotation is taken to mean flow oriented in the opposite direction to the plasma current. The relationship between $\delta W$ and sheared toroidal flow is in excellent qualitative agreement with analytic calculation of $\delta W_{h}$ for a circular large aspect ratio plasma, as shown in figure 6 of [8].

Conservation of the third adiabatic invariant, $\Phi_{\mathrm{ad}}$ - which produces strong stabilization from trapped fast particles [9]is only obtained [8] when

$$
\left\langle\omega_{\mathrm{d} h}\right\rangle+\Delta \Omega-\tilde{\omega} \gg 0,
$$

where $\left\langle\omega_{\mathrm{d} h}\right\rangle$ is the bounce-averaged hot particle toroidal drift precession frequency, $\Delta \Omega=\Omega_{E}(r)-\Omega_{E}\left(r_{1}\right)$ is the sheared toroidal flow and $\tilde{\omega}$ is the Doppler shifted mode frequency, $\tilde{\omega}=\omega-\Omega_{E}\left(r_{1}\right)$. In tokamak plasmas $\Phi_{\mathrm{ad}}$ corresponds to the flux of the poloidal magnetic field through the area defined by the toroidal precession of the trapped particle orbit centres [9]. In order to try to conserve $\Phi_{\text {ad }}$ the particles take energy from the wave and so stabilize the kink mode. Since this condition is more readily satisfied for co-rotation $\left(\Delta \Omega_{\phi}>0\right)$, co-rotating plasmas with velocity shear support more effective stabilization of the kink mode. When $\Delta \Omega_{\phi}>0$, then equation (13) can be satisfied for a smaller value of $\left\langle\omega_{\mathrm{d} h}\right\rangle$, meaning that stabilization arises from the presence of forward-precessing ions with lower energy. Since there are typically many more of these lower energy ions in a slowing down beam distribution, the stabilizing effect is enhanced. Conversely, the stabilization is diminished in counter-rotating plasmas $\left(\Delta \Omega_{\phi}<0\right)$ since $\Phi_{a d}$-conservation is inhibited, and the stabilizing contribution can come only from the less numerous higher energy ions.

The perturbed fast ion distribution function, $\delta f$, can be separated into a non-adiabatic (kinetic) part, $\delta f_{h k}$, and an adiabatic (fluid) part, $\delta f_{h f}$. This is then used to find the change in the mode energy according to equation (9). Analytic theory developed for large aspect ratio circular plasmas [8] gives the contributions to the perturbed distribution function as

$$
\begin{aligned}
\delta f_{h k}= & \sum_{l=-\infty}^{\infty} \frac{\tilde{\omega}-\Delta \Omega-n \omega_{* h}}{\tilde{\omega}-\Delta \Omega-n\langle\dot{\zeta}\rangle+l \omega_{b}} \frac{\partial f_{h}}{\partial \mathcal{E}^{0}} \\
& \times\left\langle\left(v_{\|}^{2}+\frac{v_{\perp}^{2}}{2}\right) \vec{\kappa} \cdot \vec{\xi}_{\perp} \mathrm{e}^{-\mathrm{i}\left(\omega+l \omega_{b}+n\langle\dot{\zeta}\rangle\right) t}\right\rangle
\end{aligned}
$$

and $\delta f_{h f}=-\left(Z e / M_{h}\right) \vec{\xi} \cdot \vec{\nabla} \psi_{p} \partial f_{h} / \partial \mathcal{P}_{\zeta}^{0}$, where $\omega_{* h}=$ $\left(\partial f_{h} / \partial \mathcal{P}_{\zeta}^{0}\right) /\left(\partial f_{h} / \partial \mathcal{E}^{0}\right)$ is the hot ion diamagnetic frequency, $\zeta$ is the toroidal angle, $\omega_{b}=2 \pi / \tau_{b}$ and $\tau_{b}$ is the poloidal orbit transit time. At very large flows, equation (14) tends to an asymptotic limit, since $\Delta \Omega_{\phi}$ dominates both numerator and denominator. This is evident in the behaviour of $\Re e\left(\delta W_{h}\right)$ in the large flow shear limit in figure 9.

The plasma flow will only influence mode stability when $\left|\Delta \Omega_{\phi}\right| \sim \omega_{* \mathrm{i}}$. As such it is the collisionless response of the low energy ions that is significantly modified by rotation. In typical JET plasmas, $\left|\Delta \Omega_{\phi}\right| / \omega_{* \mathrm{i}} \approx \pm 3$, so the sheared flow can strongly influence the kinetic stability of the internal kink mode. In ITER, the low rotation anticipated means that the condition for sheared flow to influence stability is unlikely to be met. 


\section{Modelling sawtooth stability in ASDEX Upgrade off-axis NBI-heated plasmas}

In order to understand the sawtooth behaviour in the ASDEX Upgrade discharges presented in section 2.2, the interaction of the MHD and fast particle effects must be considered, together with the changes in the magnetic shear. We present an explanation of these results by studying MHD stability in the presence of toroidal flows, and combining this with the effects of anisotropic hot ion distributions, as outlined in section 3. The change in the energy of the mode, $\delta W$, is then considered in relation to the sawtooth crash trigger criteria developed in [3].

In order to understand the competing physical mechanisms that underlie the sawtooth behaviour exhibited in discharges 23476 and 23477 (details of which were given in section 2.2), we have calculated the kink mode energy in the presence of both toroidal rotation and fast particles when the $q=1$ surface is moved. In the experiment, the toroidal field is raised by $\sim 10 \%$ in order to move the $q=1$ radius inwards. Similarly, in the modelling, the safety factor at the magnetic axis, $q_{0}$, is scaled, which in the MishKA-F code is achieved by scaling the toroidal field.

Equilibria have been reconstructed for discharges 23476 and 23477 at $t=3.4 \mathrm{~s}$ and $t=3.6 \mathrm{~s}$, before and after the application of off-axis NBI respectively. The equilibria are reconstructed by supplying the pressure and current profiles together with the plasma shape to the HeLENA code [30]. Whilst Helena is a fixed-boundary code solving the static GradShafranov equation so that it neglects the toroidal rotation, the effect of the flow on the equilibrium is expected to be negligible at such low injected beam powers. The current profile is found from the Transp code [26]. The pressure profile is derived from diagnostic measurements; the electron temperature is found from the Thomson scattering diagnostic and the electron density is found by fitting a modified hyperbolic tangent to the the core Thomson scattering data and the lithium beam data at the pedestal. Upon supplying these profiles to HelenA, the equilibrium is further constrained so that the $q=1$ surface is matched to the inversion radius found from the soft x-ray diagnostic and the plasma pressure is scaled so that $\beta_{\mathrm{p}}=p /\left(B_{a}^{2} / 2 \mu_{0}\right)$ is as found by TRANSP, where $B_{a}=\mu_{0} I / l$ and $l$ is the poloidal perimeter of the plasma. It is necessary to constrain the $q$-profile appropriately since the effect of the energetic passing ions is determined by the ions with orbits that cut the $q=1$ surface [7].

In order to retain the complex dependence of the fast ion population upon pitch angle, energy and radius, the full Monte-Carlo distribution function is employed in the HAGIS drift kinetic modelling. In both discharges, the energetic particles are peaked at $\lambda \sim 0.5$ with an approximately Gaussian dependence. At lower energy, the beam ions have a more isotropic dependence. Shot 23477 has slightly improved confinement and a higher passing fraction. When the ions are injected tangentially, the higher toroidal field means that the birth pitch angle is more aligned to the field lines, and so it takes longer for the distribution to spread in pitch angle space. Figure 10 shows the dependence of the fast ion source rate upon the radius as calculated by TRANSP. In both 23476 and 23477 the peak of the NBI fast ion population is outside the $q=1$ radius.

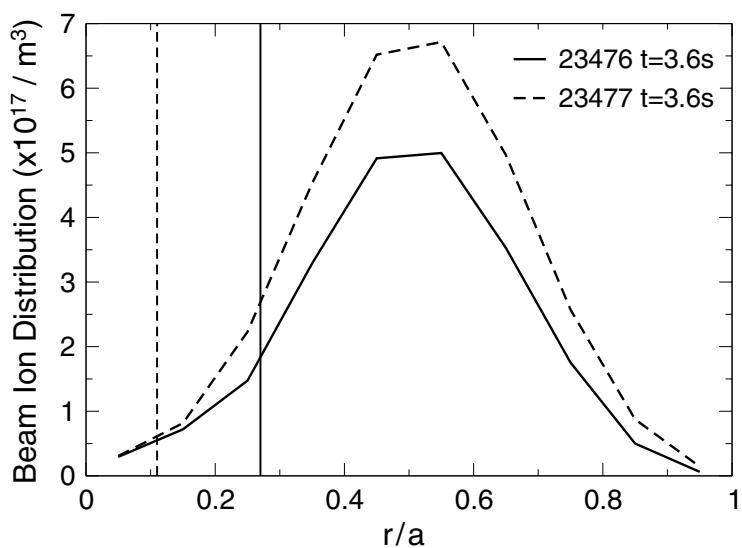

Figure 10. The distribution function of the beam fast ions in ASDEX Upgrade as a function of radius. In both shots 23476 and 23477 , the peak of the fast ion population is outside the $q=1$ radius, marked by the vertical lines.

The equilibrium reconstructed in accordance with the procedure outlined above is unstable to the ideal $n=1$ internal kink mode at the experimental $q=1$ radii. In discharge 23476, the $q=1$ surface is approximated using the inversion radius on the SXR diagnostic as $\psi_{1}=0.25$, whereas in discharge 23477 it is $\psi_{1}=0.15$. As $q_{0}$ is dropped, and $\psi_{1}$ is increased, the ideal MHD stability drive increases and $\delta W_{\text {MHD }}$ decreases.

The toroidal rotation profile used is that measured by charge exchange recombination spectroscopy. However, the design of the discharge meant that the rotation profile was only measured early in the discharge and not in the off-axis NBI phase. Since this modelling only permits a qualitative discussion of the physical effects on the sawteeth, this is an allowable approximation. The important thing to note is that the toroidal rotation is expected to increase significantly when the off-axis NBI is applied. The measured rotation at the magnetic axis in the on-axis NBI phase is of the order of $80 \mathrm{~km} \mathrm{~s}^{-1}$, and the more tangential off-axis PINI could be anticipated to lead to even faster rotation. This must be compared with the preceding ICRF-only phase, where Doppler reflectometry measurements [41] in previous ASDEX Upgrade shots [42] suggest that the plasma will only rotate slowly, or perhaps, even rotate counter- $I_{\mathrm{p}}$. When the toroidal rotation is increased, the $n=1$ internal kink mode is stabilized. However, as a linear eigenvalue solver, the MishKA-F code can only find $\delta W_{\text {MHD }}$ (from normalization by the inertia term) when the mode is unstable. Thus, in order to find $\delta W_{\text {MHD }}$ in the stable regime, we have increased the plasma pressure for a given $r_{1}$ and $v_{\phi 0}$ into the region of parameter space where the kink mode is driven unstable, then extrapolated the linear dependence of $\delta W$ with $\beta_{\mathrm{N}}$ to find the change in the mode energy at the experimental plasma pressure. The fluid component of $\delta W$ for three different core velocities can be seen in figure 11, which shows that $v_{\phi 0}=50,100 \mathrm{~km} \mathrm{~s}^{-1}$ stabilizes the kink mode to a $q=1$ radius of $\psi_{1} \approx 0.2$. The step in the mode stabilization at $\psi_{1} \sim 0.18$ can be explained by the fact that increasing $\psi_{1}$ leads to the $q=1$ surface moving outside the region of strongest flow shear.

In addition to the effect of sheared flow on the fluid drive of the mode, we can assess the (de)stabilization effects from the energetic particles born due to both NBI and ICRH. The 


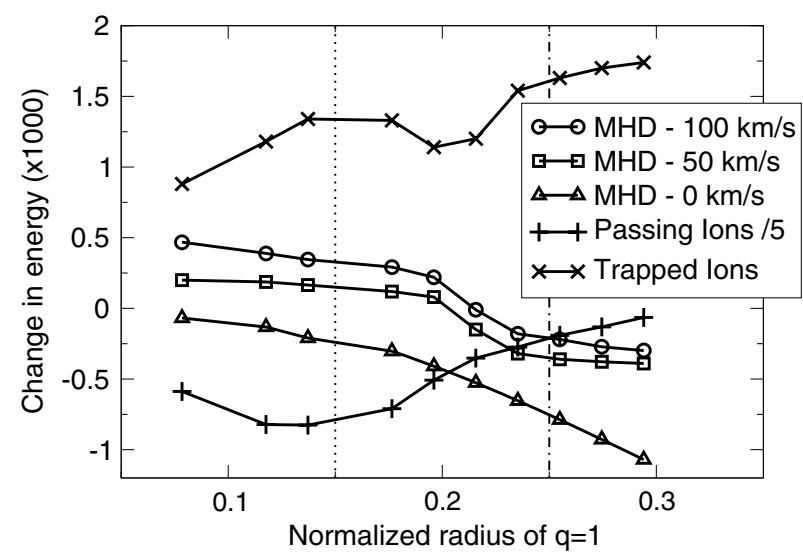

Figure 11. The components of the change in the energy of the $n=1$ internal kink mode as a function of the normalized radius of the $q=1$ surface. The passing fast ions are destabilizing and can dominate over the trapped ions born due to the NBI and ICRH. The fluid instability drive is stabilized by increasing the toroidal rotation. The MHD components of the change in mode energy are shown for different values of core toroidal rotation speed.

NBI fast ions are represented by the distribution function of markers taken directly from the Monte-Carlo solver in the TRANSP code [26]. The ICRF fast ion distribution function is represented by a bi-Maxwellian function, as given in [43] as

$$
\begin{aligned}
& f_{h}^{\mathrm{ICRH}}=\left(\frac{m}{2 \pi}\right)^{3 / 2} \frac{n_{c}(r)}{T_{\perp}(r) T_{\|}^{1 / 2}(r)} \\
& \quad \times \exp \left[-\frac{\mu B_{c}}{T_{\perp}(r)}-\frac{\left|\mathcal{E}-\mu B_{c}\right|}{T_{\|}(r)}\right],
\end{aligned}
$$

where the particle energy $\mathcal{E}=m v^{2} / 2$, the magnetic moment $\mu=m v_{\perp}^{2} / B, T(r)$ is the fast ion temperature $\|$ and $\perp$ represent the components parallel and perpendicular to the magnetic field respectively, $B_{c}$ is the field strength at the resonance and $n_{c}$ is the local density evaluated at $B=B_{c}$. Assuming a $5 \%$ hydrogen concentration, the FELICE code [44] predicts that the RF resonance will be within the $q=1$ radius in both discharges, with the resonance location in discharge 23476 on the high field side whilst in 23477 it is on the low-field side of the magnetic axis. It is also found in the HaGis modelling that the change in the mode energy due to the ICRF trapped energetic ions does not significantly alter as the $q=1$ radius is moved. This is partly because the RF ions remain largely within $q=1$ so the stabilization arising from kinetic effects does not change markedly. What small change there is in $\delta W_{h}^{t}$ is offset by the change in the magnetic shear which occurs as $q=1$ is moved inwards. Discharge 23477, which has fewer trapped ions inside $q=1$, and so a marginally reduced $\delta W_{h}^{t}$, also has a lower $s_{1}$, so in accordance with equation (1), the sawtooth trigger quotient remains largely unchanged. This is consistent with the experiment where the sawtooth period remains approximately constant when the toroidal field is increased.

As the toroidal field is scaled, and consequently the eigenfunction changes, the contribution of the fast particles to the change in the potential energy of the mode is assessed. Figure 11 shows the components of the change in the mode energy, $\int \partial \delta W / \partial r \mathrm{~d} r$, due to the trapped (both NBI and ICRH)

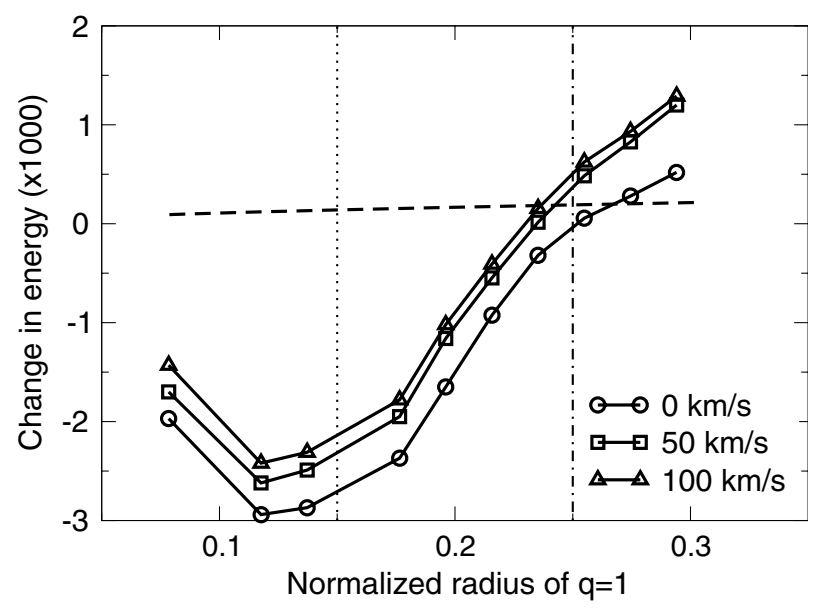

Figure 12. The total change in the energy of the internal kink mode as a function of the normalized radius of $q=1$. The mode is linearly unstable for $r_{1}=0.15$, as in discharge 23477 , and conforms to the Porcelli trigger condition. Conversely, it is marginally stable for $r_{1}=0.25$, and when rotation up to $100 \mathrm{~km} \mathrm{~s}^{-1}$ is included, the Porcelli trigger condition is not satisfied.

and passing energetic ions. It is evident that the passing fast ions are destabilizing, and furthermore that the local effects of these passing ions at $q=1$ can dominate over the effects of the trapped fast ions. Also, the difference in $\beta_{\mathrm{N}}$ exhibited experimentally between these shots cannot account for the different sawtooth behaviour since the fast ion effects dominate over the MHD drive and $\beta_{h}$ is approximately the same in the two discharges.

Interestingly, the destabilization arising from the passing ions is not maximized at the radial location at which the positive fast ion pressure gradient is maximum. The most unstable location is somewhat inside the point at which $\nabla P_{h}$ is maximum. One possible explanation for this is that $\delta W_{h}$ is normalized by $\epsilon_{1}^{2}=r_{1}^{2} / R_{0}^{2}$, meaning that the narrower $q=1$ radius accentuates the normalized change in the potential energy [7]. Furthermore, the finite orbit width of the passing ions means that the biggest contribution of the ions that cross the $q=1$ surface need not necessarily be at the region of the steepest gradient of the hot particle pressure. As $\psi_{1}$ is increased, the passing ions become less destabilizing, and are even stabilizing as $\psi_{1}>0.34$, which happens when the $q=1$ surface approaches the peak of the off-axis distribution function.

Figure 12 shows the total change in the mode energy with respect to the radial location of $q=1$ at different core toroidal velocities. The mode energy is appropriately normalized and compared with the Porcelli crash trigger condition $\left(s_{1} \rho / r_{1}=\right.$ $\rho \mathrm{d} q / \mathrm{d} r(q=1))$, given in equation (1). In a static equilibrium, the Porcelli model [3] predicts that the kink mode is unstable at both $\psi_{1}=0.15$ (discharge 23477 , indicated by the dashed line) and $\psi_{1}=0.25$ (shot 23476, dotted-dashed line). In order to include the effect of rotation, not only is the rotation included in the MHD stability analysis, as illustrated in figure 11, but the effect of the flow shear is included in the trapped fast ion response [40]. Whilst it has been shown in section 3.4 that flow shear can significantly affect the role of the trapped ions, in these ASDEX Upgrade discharges it results in only a mild change in $\delta W_{h}^{t}$. It is evident that increasing the toroidal 
rotation $\left(\sim 100 \mathrm{~km} \mathrm{~s}^{-1}\right.$ could be anticipated in these shots) results in a stabilization of the mode, and the crash trigger condition is no longer met at $\psi_{1}=0.25$. This modelling suggests that the increase in sawtooth period in discharge 23476 when the off-axis NBI is switched on is explained by the significant increase in toroidal rotation arising from the tangential PINI. Conversely, whilst there is also an increase in rotation in discharge 23477, now the $q=1$ surface is sufficiently core localized that the destabilization from the passing energetic ions is able to overcome the stabilization from the mode, and shorter sawteeth could be expected. This is not inconsistent with the experiment, where no clear sawteeth are observed, though small, frequent oscillations are one plausible interpretation.

This modelling suggests that the passing fast ions do play an important role in determining the sawtooth behaviour in these ASDEX Upgrade plasmas. Furthermore, this has important implications for the use of off-axis NBI for sawtooth control. Whilst the passing ions are destabilizing when injected outside the $q=1$ radius, the peak of the deposition of the beam ions must be located sufficiently far outside $q=1$ for this destabilization to trigger sawteeth. This effect is not as localized as that from ICRH [18], but the magnitude of the destabilization is not as large either. Another important consideration for the application of off-axis NBI is the role of the toroidal rotation. When the neutral beams are injected offaxis this results in an enhanced spin up of the plasma, which generally has a stabilizing effect on the internal kink mode, competing with the destabilizing kinetic effects. Since the rotation in ITER plasmas is likely to be very small, the stability modelling of off-axis NBI-heated static plasmas in [5] would not be significantly affected.

As well as changing the mode potential energy, another possible way to influence the sawtooth behaviour is to increase the magnetic shear at $q=1$, which is apparent in equation (1). The application of neutral beam injection does also drive current, which has been shown to be significant in ASDEX Upgrade plasmas under certain plasma conditions [45, 46]. In previous studies in ASDEX Upgrade using off-axis NBI, the change in the sawtooth behaviour had been explained by the neutral beam current drive [11]. In this case, the on-axis source led to sawteeth with a period of approximately $\tau_{\mathrm{s}} \sim 60 \mathrm{~ms}$. Meanwhile, one off-axis PINI (source 7) led to approximately the same sawtooth period, $\tau_{\mathrm{s}} \sim 60 \mathrm{~ms}$, whilst the most tangential off-axis PINI (source 6 ) gave $\tau_{\mathrm{s}} \sim$ $200 \mathrm{~ms}$. Subsequent TRANSP runs have found that whilst there is a small perturbation to the current profile due to NBCD, the magnetic shear at the $q=1$ surface is practically indistinguishable for the two off-axis sources, making it hard to explain such a marked change in behaviour through the driven current. Further, the neutral beam current drive in previous experiments matches the TRANSP modelling well at low power [45], so the prediction of a negligible change to $s_{1}$ seems reliable. All the sources lead to predominantly passing fast ion populations. These passing fast ions can be destabilizing when the co-passing fast particle pressure gradient at the $q=1$ surface is positive. This is indeed the case for source 7 , explaining the relatively small sawteeth. Conversely, whilst the fast ions from source 6 are born outside $q=1$, they are so far off-axis that the passing fast ions do not strongly influence the kink mode stability. Furthermore, the tangential beams can lead to an increased spin up of the plasma, leading to a stronger toroidal rotation stabilization of the kink mode, perhaps responsible for the long sawtooth period. In the previous experiments [11] the effect of the rotation may have been more significant than the discharges reported here since the plasmas were at lower density and lower current, leading to an enhanced momentum input from higher first orbit losses.

\section{Discussion of sawtooth control mechanisms}

Comprehensive numerical modelling has been employed to quantify the relative roles of fast anisotropic ions, the toroidal rotation and changes to the magnetic shear profile in determining sawtooth behaviour. The control of sawteeth is important for baseline scenario operation of burning plasmas, since plasmas with long sawtooth periods are more susceptible to NTMs, resulting in substantial confinement degradation. The stabilizing effects of alpha particles are likely to exacerbate this, so it should be of high priority for current tokamaks to demonstrate that long-period sawteeth can be reliably ameliorated, whether it be by ECCD, ICRH or off-axis NBI.

Recent modelling [47] showed that the ECCD planned as the primary sawtooth control actuator in ITER is theoretically capable of modifying the magnetic shear to a sufficient extent as to destabilize sawteeth which have been stabilized by the fusion-born $\alpha$ particles. However, as yet, there has been no experimental verification that ECCD can destabilize long fast ion induced sawteeth. Initial results from TORE-SUPRA [15] are promising, though they do exhibit some unexplained behaviour.

Whilst off-axis NBI can in theory result in a destabilization of the long sawteeth expected in ITER, it has been shown [5] that the beam passing ions will strongly stabilize the internal kink mode unless the $q=1$ surface is very core localized, $r_{1}<0.2$. In the baseline scenario in ITER the $q=1$ surface is expected to be sufficiently broad as to approach mid-radius, so the NBI ions are likely to be strongly stabilizing, even if injected off-axis [25]. Thus modelling to predict the efficacy of the ECCD system ought to include the role of the N-NBI passing ions.

Counter propagating waves could be more effective than off-axis N-NBI since they are highly energetic and have strong radial shears in the parallel asymmetries of the distribution function, accentuating the destabilization term arising from the presence of energetic passing ions. In recent JET discharges [48] it has been shown that a change in the magnetic field of only about two percent can be sufficient to enable or disable sawtooth control. The corresponding change in the magnetic shear has been calculated, and was shown to be extremely modest, thus questioning the viability of the classical sawtooth control mechanism relating to the change in the magnetic shear due to ICCD [17]. Nevertheless, it has been shown that when a counter propagating wave is deposited sufficiently accurately on the high field side, the fast ion effect is so strong that the internal kink mode is driven not only resistive unstable, but ideally unstable, and this in turn is consistent with measured sawteeth that are much shorter in period than those obtained in Ohmic plasmas [18]. Furthermore, unlike the classical sawtooth control mechanism, the fast ion mechanism 
is independent of the electron drag, which is expected [49] to limit the ICCD current drive efficiency of the proposed ICRF system for ITER.

\section{Conclusions}

We have investigated sawtooth behaviour in off-axis neutral beam heated plasmas in JET and ASDEX Upgrade. It is found that the energetic particles born outside the $q=1$ surface due to the NBI can destabilize the sawtooth oscillations, even in the presence of stabilizing on-axis fast ions. The effect of the passing fast ions has been modelled using the drift kinetic HAGIS code and found to be significant. Furthermore, the passing particle stabilization mechanism has been considered, and found to be attributable to the contribution of adiabatic ions that intersect the $q=1$ surface. We have also considered the effect of equilibrium flow shear on the stabilization arising from the presence of fast trapped ions. All of these effects are then included in modelling to assess the sawtooth stability in ASDEX Upgrade plasmas. We find that whilst the off-axis passing ions can be destabilizing, the enhanced toroidal rotation from injecting off-axis NBI is stabilizing, and the two mechanisms compete to give rise to the experimental results presented here. It should be noted that no direct comparison can be drawn between the modelling and the experimental results since the sawtooth behaviour is inherently non-linear, whereas here we consider the linear stability of the $n=1 \mathrm{kink}$ mode. This analysis shows that both the passing fast particles and the toroidal flow shear are significant in determining the kink mode stability in the presence of an energetic particle population.

\section{Euratom (c) 2009.}

\section{Acknowledgments}

This work was partly funded by the United Kingdom Engineering and Physical Sciences Research Council and by the European Communities under the contract of Association between EURATOM and UKAEA. The views and opinions expressed herein do not necessarily reflect those of the European Commission. The work was partly carried out within the framework of the European Fusion Development Agreement.

\section{References}

[1] Sauter O. et al 2002 Phys. Rev. Lett. 88105001

[2] Gude A., Günter S., Maraschek M. and Zohm H. 2002 Nucl. Fusion 42833

[3] Porcelli F., Boucher D. and Rosenbluth M. 1996 Plasma Phys. Control. Fusion 382163

[4] Hu B., Betti R. and Manickam J. 2006 Phys. Plasmas 13112505

[5] Chapman I.T., Jenkins I., Budny R.V., Graves J.P., Pinches S.D. and Saarelma S. 2008 Plasma Phys. Control. Fusion 50045006

[6] Nave M.F.F. et al 2003 Nucl. Fusion 431204

[7] Graves J.P. 2004 Phys. Rev. Lett. 92185003

[8] Graves J.P., Sauter O. and Gorelenkov N. 2003 Phys. Plasmas 101034
[9] Porcelli F. 1991 Plasma Phys. Control. Fusion 331601

[10] Kruskal M. and Oberman C. 1958 Phys. Fluids 1275

[11] Mück A., Goodman T.P., Maraschek M., Pereverez G., Ryter F. and Zohm H. 2005 Plasma Phys. Control. Fusion 471633

[12] Angioni C., Goodman T., Henderson M. and Sauter O. 2003 Nucl. Fusion 43455

[13] Ikeda Y. et al 2002 Nucl. Fusion 42375

[14] Westerhof E. et al 2006 Proc 14th Joint Workshop on Electron Cyclotron Emission and Electron Cyclotron Heating (Santorini, Greece, Heliotopos Conferences Ltd) p 38

[15] Lennholm M. et al 2008 15th Joint Workshop on ECE and ECRH (California, USA)

[16] Eriksson L.G. et al 2004 Phys. Rev. Lett. 92235004

[17] Eriksson L.G. et al 2006 Nucl. Fusion 46 S951

[18] Graves J.P., Chapman I.T., Coda S., Eriksson L.G. and Johnson T. 2009 Phys. Rev. Lett. 102065005

[19] Nave M.F.F. et al 2006 Phys. Plasmas 13014503

[20] Chapman I.T., Pinches S.D., Graves J.P., Appel L.C., Hastie R.J., Hender T.C., Saarelma S., Sharapov S.E. and Voitsekhovitch I. 2007 Phys. Plasmas 14070703

[21] Chapman I.T., Hender T.C., Saarelma S., Sharapov S.E., Akers R.J., Conway N.J. and the MAST Team 2006 Nucl. Fusion 461009

[22] Chapman I.T., Pinches S.D., de Bock M., Krämer-Flecken A., Koslowski H.R. and Liang Y. 2008 Nucl. Fusion 48035004

[23] Kramer G.J. et al 2000 Nucl. Fusion 401383

[24] Graves J.P. et al 2005 Plasma Phys. Control. Fusion 47 B121

[25] Chapman I.T. et al 2007 Plasma Phys. Control. Fusion 49 B385

[26] Budny R.V. et al 1992 Nucl. Fusion 32429

[27] ITER Technical Basis for Final Design 2001 ITER Documentation Series \#24 (Vienna: IAEA) p 2 (chapter 2.5)

[28] Waelbroeck F.L. 1996 Phys. Plasmas 31047

[29] Chapman I.T., Huysmans G.T.A., Mikhailovskii A.B. and Sharapov S.E. 2006 Phys. Plasmas 13065211

[30] Huysmans G., Goedbloed J. and Kerner W. 1991 Proc. CP90 Conf. on Computational Physics Proc. (Amsterdam, The Netherlands) (Singapore: World Scientific) p 371

[31] Wahlberg C. and Bondeson A. 2000 Phys. Plasmas 7923

[32] Pinches S.P. et al 1998 Comput. Phys. Commun. 111133 (Release Version 8.09)

[33] Madden N. and Hastie R.J. 1994 Nucl Fusion 34519

[34] Briezman B., Candy J., Porcelli F. and Berk H. 1998 Phys. Plasmas 52326

[35] Porcelli F., Stankiewicz R., Kerner W. and Berk H. 1994 Phys. Plasmas 1470

[36] Mikhailovskii A.B. and Skovoroda A.A. 2002 Plasma Phys. Control. Fusion 442033

[37] Angioni C. et al 2002 Plasma Phys. Control. Fusion 44205

[38] Kolesnichenko Ya., Marchenko V.S. and White R.B. 2005 Phys. Plasmas 12022501

[39] Wang S., Ozeki T. and Tobita K. 2002 Phys. Rev. Lett. 88105004

[40] Graves J.P., Hastie R.J. and Hopcraft K. 2000 Plasma Phys. Control. Fusion 421049

[41] Conway G.D. et al 2004 Plasma Phys. Control. Fusion 46951

[42] Conway G.D. et al 2006 Nucl. Fusion 46 S799

[43] Graves J.P., Cooper W.A., Coda S., Eriksson L.G. and Johnson T. 2006 Proc Varenna-Lausanne Theory of Fusion Plasmas (Varenna, Italy) vol 871 (New York: AIP) p 350

[44] Brambilla M. and Krucken T. 1988 Nucl. Fusion 281813

[45] Günter S. et al 2006 Proc. 21st Int. Conf. on Fusion Energy (Chengdu, China) (Vienna: IAEA) EX6-1

[46] Günter S. et al 2005 Nucl. Fusion 45 S98

[47] Henderson M. et al 2005 3rd IAEA TM on ECRH Physics and Technology in ITER (Como, Italy) p 143

[48] Coda S. et al 2007 Proc 34th EPS Conf. on Plasma Physics (Warsaw, Poland) P5.130

[49] Laxåback M. and Hellsten T. 2005 Nucl. Fusion 451510 